\title{
Estudio de dos métodos pulvimetalúrgicos empleados en la fabricación de biomateriales compuestos de titanio e hidroxiapatita.
}

\section{Study of two powder metallurgical methods used in composed biomaterials manufacturing of titanium and hydroxyapatite.}

Presentación: 6-7/10/2020

\section{Doctorando:}

\section{César Andrés Roure}

Laboratorio del Dpto. de Ingeniería Metalúrgica, Facultad Regional Córdoba, Universidad Tecnológica Nacional - Argentina cesararoure@hotmail.com.ar

\section{Director/es:}

\section{Carlos Rodolfo Oldani}

\section{Co-director/es:}

\section{Roberto Oscar Lucci}

\section{Resumen}

En el siguiente trabajo se plantea un análisis comparativo entre dos metodologías de fabricación que emplean métodos pulvimetalúrgicos que permiten obtener piezas sinterizadas base titanio, con agregado de hidroxiapatita como material bioactivo. El primer método utiliza técnicas tradicionales de obtención de muestras por compactación de polvos a través de prensas y matricería metálica. El segundo método emplea la técnica de gel-casting combinada con la tecnología de impresión 3D para la fabricación de moldes. Se lleva a cabo un análisis de los métodos de manufactura, la tecnología empleada en el desarrollo de moldes, las variables de sinterización y las propiedades mecánicas, tales como el módulo de elasticidad, resistencia mecánica, límite de fluencia, elongación y dureza de los materiales de titanio e hidroxiapatita obtenidos.

Palabras clave: Titanio, Hidroxiapatita, Gel-casting, Compactación, Sinterización, Propiedades Mecánicas.

\begin{abstract}
The following work presents a comparative analysis between two manufacturing methodologies that use powder metallurgical methods, that allow obtaining titanium-based sintered parts with the addition of hydroxyapatite as a bioactive material. The first method uses traditional techniques for obtaining samples by powders compaction through presses and metal molds. The second method employs the gel-casting technique combined with 3D printing technology for mold making. An analysis of the manufacturing methods, the technology used in the development of molds, the sintering variables and the mechanical properties about titanium and hydroxyapatite's samples is carried. Some of the mechanical properties that will be analyzed are the modulus of elasticity, mechanical resistance, yield point, elongation and hardness.
\end{abstract}

Keywords: Titanium, Hidroxyapatite, Gel-casting, Compaction, Sintering, Mechanical Properties. 


\section{Introducción}

Un biomaterial se implanta con el objeto de sustituir o regenerar tejidos que han sufrido daños o se han perdido por traumatismos, malformaciones o enfermedades degenerativas. La principal problemática en la sustitución de una prótesis está en que el hueso receptor se encuentre más deteriorado. Por ello, es importante desarrollar nuevos materiales y tratamientos superficiales que aumenten la vida útil, mejoren la biocompatibilidad y pospongan las revisiones (Lario-Femenía et al., 2016).

Según su composición química, los biomateriales se clasifican en metálicos, poliméricos, cerámicos y compuestos; de acuerdo con su origen en naturales y sintéticos y por su estructura, en compactos y poroso. Analizando el caso de los biomateriales metálicos, el primer requisito para su utilización es que sean tolerados por el organismo. Otro requisito también imprescindible es que tengan una buena resistencia a la corrosión (Duffo, 2011). Un ejemplo de biomaterial metálico es el titanio que presenta una gran resistencia a la corrosión, capaz de resistir el ataque de la mayoría de los ácidos orgánicos y de soluciones de cloro. La capa de óxido que crece espontáneamente sobre este, es la responsable de las propiedades contra la corrosión en su superficie. Se ha comprobado que el titanio y sus aleaciones consiguen una interfase equivalente a la osteointegración en los implantes usados en seres humanos. Si mencionamos sus propiedades mecánicas, el titanio presenta valores para su módulo de elasticidad que se encuentra más próximo al del hueso que el resto de los metales. Las aleaciones comerciales de titanio, con una pureza del 99,2 \%, tienen una tensión de rotura de unos $434 \mathrm{MPa}$ (Padilla et al., 2018; Traini et al., 2015).

Por otra parte, para los biomateriales cerámicos, su principal ventaja es su baja reactividad química que conlleva una clara biocompatibilidad, y con la posibilidad de también ser bioactivos. Sin embargo, la fragilidad de las biocerámicas restringió su campo de aplicación. El fosfato de calcio (hidroxiapatita) es utilizado en la fabricación de implantes por presentar buena biocompatibilidad, bioactividad, osteoconductividad y unión directa al hueso (GarcíaGarduño \& Reyes-Gasga, 2006). Estudios in vitro indicaron que la HAP es un fuerte estimulador del ligamento periodontal y que, además, actúa como un estimulador de la proliferación celular (Pereira G \& Oliva M, 2014). El principal inconveniente de la hidroxiapatita radica en sus propiedades mecánicas deficientes. La HAP policristalina tiene un módulo elástico elevado respecto al hueso, entre 40 y 117 GPa (Londoño et al., 2006).

Una alternativa viable es el uso de biomateriales compuestos, de manera que obtenga un nuevo material con propiedades óptimas en aplicaciones biomédicas. Tal es el caso de la combinación del titanio, que aporta buenas propiedades mecánicas, bajo módulo de Young y un comportamiento bioinerte, con otro material como es la hidroxiapatita que promueve la osteointegración (Niespodziana et al., 2010).

Por otra parte, se ha comprobado que el uso de estructuras porosa reduce eficientemente el valor del módulo elástico, lo que beneficiaría que estos valores para el titanio se aproximen al del hueso. Además, en el caso de aplicaciones en implantes, promueve el crecimiento óseo, gracias a la capacidad de vascularización y aumento del área de fijación en la interfaz implante-hueso (Segura \& Soria, 2019).

Dentro de las tecnologías que permiten fabricar materiales compuestos de titanio (Ti) e hidroxiapatita (HAP), podemos mencionar a la pulvimetalurgia -powder metallurgy- (PM). La PM tradicional consiste en el uso de una matriz metálica, la cual es rellenada con los materiales formadores del compuesto, y a través de una prensa, comprimir dichos polvos en la geometría del molde, con un posterior sinterizado que permita la difusión en las partículas metálicas/cerámicas (Bocco \& Colazo, 2017). Otra de las alternativas de fabricación por pulvimetalurgia es el proceso de gel-casting, donde a partir de una suspensión de polvos metálicos/cerámicos y resina acrílica (RA), es vertida y secada en un molde. La muestra obtenida (pieza "en verde"), luego es sinterizada, dando como resultado una pieza compuesta de matriz metálica (Roure \& Cantero, 2016).

Por otro lado, la incorporación de la tecnología de impresión 3D abre una gran puerta a nuevos métodos de fabricación de prótesis, ya que permite obtener implantes altamente personalizadas a través del diseño por CAD-CAM y con geometrías complejas y terminación superficial controlada, permitiendo así, poder fabricar moldes aptos a las necesidades de los usuarios (Horvath \& Cameron, 2017; Suárez Castrillón et al., 2015).

De esta manera, se espera determinar las diferencias en el empleo de los métodos pulvimetalúrgicos mencionados, que permiten fabricar biomateriales compuestos metal-cerámico con titanio-hidroxiapatita, analizando la tecnología empleada en el desarrollo de moldes, las variables de sinterización y las propiedades mecánicas. 


\section{Desarrollo}

\section{Materiales y Métodos}

Para realizar este estudio, se fabricaron muestras a partir de dos métodos pulvimetalúrgicos que utilizan material en polvo como materia prima. La Figura 1 muestra los diagramas de flujo que describen ambos procesos pulvimetalúrgicos. La Figura 1a describe el método que emplea compactación tradicional (CT), el cual utiliza los polvos de Ti e HAP que se estudian, en mezclas de diferentes proporciones en peso y luego son compactados con una prensa hidráulica, en una matriz metálica de geometría cilíndrica. Por último, los compactos en verde obtenidos se sinterizan en horno con atmósfera de vacío. La Figura $1 \mathrm{~b}$ esquematiza el método pulvimetalúrgico que utiliza la técnica de preparación por gel-casting y obtención de muestras en moldes fabricados por impresión 3D en polímeros (GC3D). Este método utiliza polvos de Ti, HAP y RA en diferentes proporciones en peso que luego son aglutinados por un proceso de polimerización en moldes impresos con tecnología 3D. Las muestras en verde se extraen del molde y son sinterizadas en horno con protección de atmósfera en alto vacío.

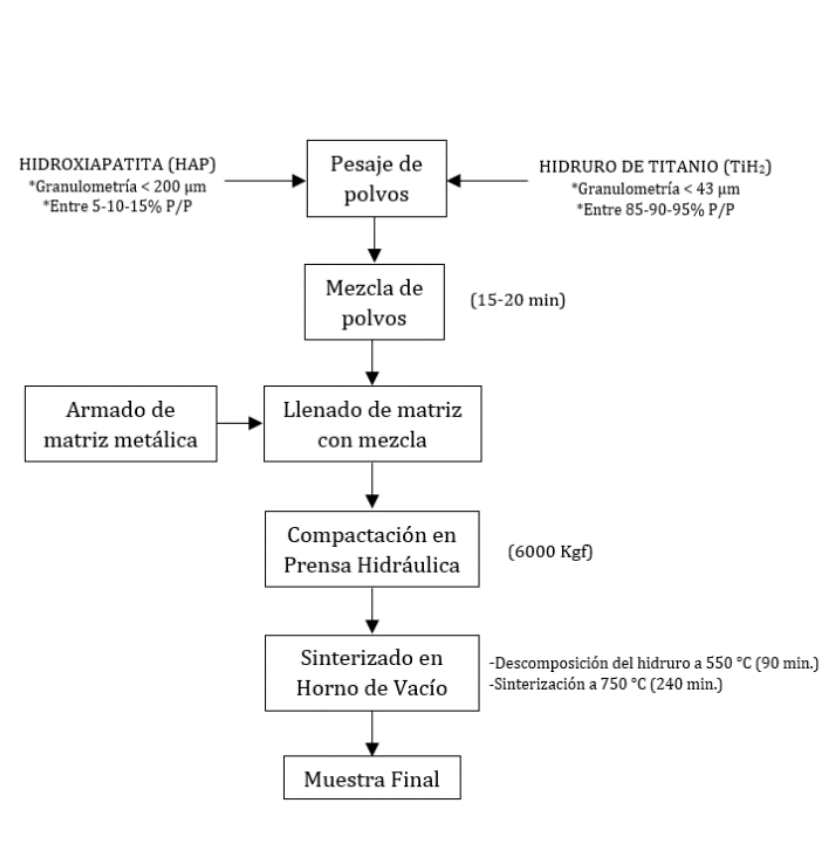

(a)

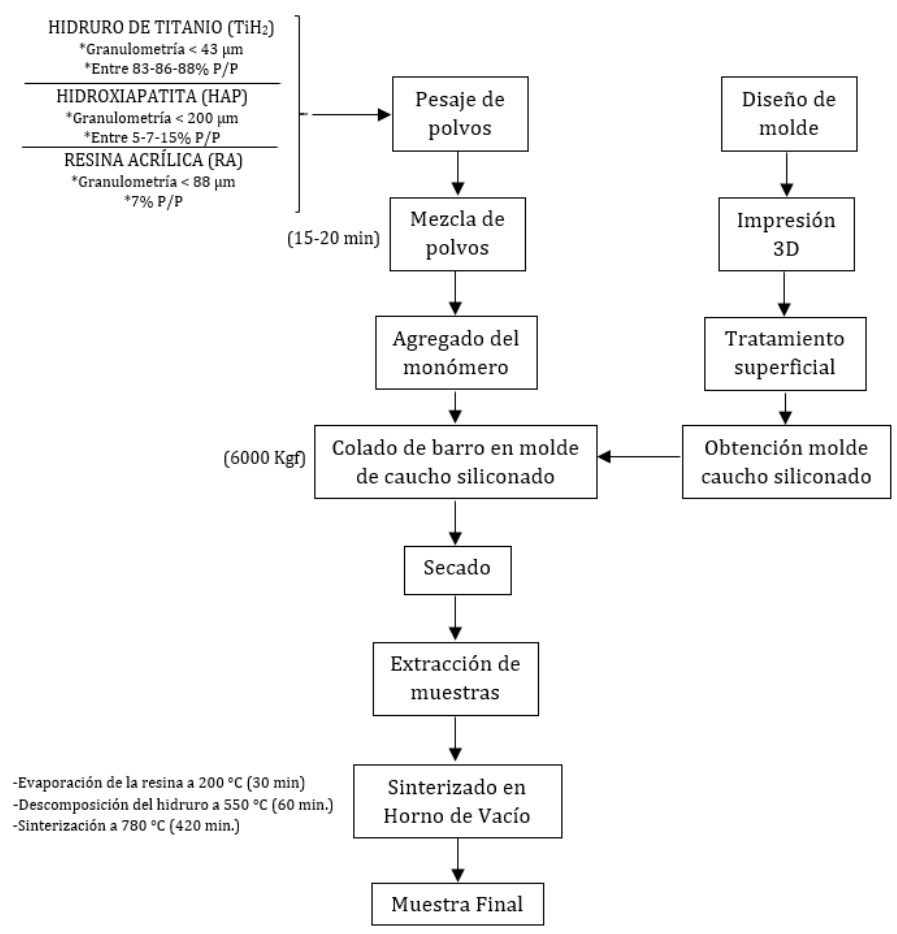

(b)

Figura 1. Diagramas de flujo de los métodos pulvimetalúrgicos estudiados. (a) Compactación tradicional (CT). (b) Gel-casting e impresión 3D (GC3D).

La Tabla 1 detalla las concentraciones de polvos empleadas para cada método pulvimetalúrgico. Para el método CT se emplea polvo de hidruro de titanio (II) de la compañía "Sigma Aldrich", tamaño de partícula inferior a los $44 \mu \mathrm{m}$ y pureza del $98 \%$; mezclada con hidroxiapatita en polvo de origen bovino (marca Tissum) con una granulometría menor a $200 \mu \mathrm{m}$. Las proporciones de las mezclas que se estudian son con 5, 10 y $15 \%$ en peso de hidroxiapatita, y el restante formado por hidruro de titanio (II). La mezcla de polvos se realiza en un mezclador de vidrio tipo "Y" durante un tiempo de 15 minutos a $34 \mathrm{rpm}$. Posteriormente, la mezcla es vertida en el interior de una matriz metálica con forma cilíndrica de $8 \mathrm{~mm}$ de diámetro y $8 \mathrm{~mm}$ de altura, realizando su compactación mecánica por simple efecto mediante prensado hidráulico. Para ello, se utiliza una carga de compactación de $590 \mathrm{MPa}$, lo cual garantiza la obtención de muestras en verde con la suficiente resistencia mecánica para su manipulación previa a la etapa de sinterización.

El método GC3D emplea polvo de hidruro de titanio (II) ( $44 \mu \mathrm{m}$ ) de la compañía "Sigma Aldrich", polvos de resina acrílica compuestos por metacrilato de polimetilo $(88 \mu \mathrm{m})$ de la compañía "Cardent" e hidroxiapatita en polvo de origen sintético de la compañía "Sigma Aldrich". Al igual que el método CT, la mezcla de polvos se realiza con un mezclador de vidrio tipo "Y" durante un tiempo de 15 minutos a $34 \mathrm{rpm}$. Se estudian mezclas con cantidades fijas de $7 \%$ de RA y cantidades de 5, 7 y $10 \%$ de HAP. Posteriormente, la mezcla de estos polvos es combinada con peróxido de benzoilo (iniciador de reacción) y metacrilato de metilo (monómero de secado automático), ambos en estado líquido que permiten obtener una solución viscosa o pasta, conocida como lodo o "slurry". Cuando la pasta consigue una viscosidad con suficiente fluidez, es vertida dentro de un molde de $7 \mathrm{~mm}$ de diámetro y $12 \mathrm{~mm}$ de altura, obtenido 
con la asistencia de la tecnología de impresión 3D que permite extraer las muestras en verde después de 24 hs de secado.

Tabla 1. Concentraciones de las mezclas y designación de las muestras para cada método pulvimetalúrgico.

\begin{tabular}{|c|c|c|c|c|}
\hline Método & $\begin{array}{c}\text { Designación de } \\
\text { la muestra }\end{array}$ & $\begin{array}{c}\text { Concentración de } \\
\mathbf{T i H}_{\mathbf{2}}[\% \text { P/P] }\end{array}$ & $\begin{array}{c}\text { Concentración de } \\
\text { HAP [\% P/P] }\end{array}$ & $\begin{array}{c}\text { Concentración de } \\
\text { RA [\% P/P] }\end{array}$ \\
\hline \multirow{3}{*}{ CT } & $95-5$ & 95 & 5 & - \\
\cline { 2 - 5 } & $90-10$ & 90 & 10 & - \\
\cline { 2 - 5 } & $85-15$ & 85 & 15 & - \\
\hline \multirow{3}{*}{ GC3D } & $88-7-5$ & 88 & 5 & 7 \\
\cline { 2 - 5 } & $86-7-7$ & 86 & 7 & 7 \\
\cline { 2 - 5 } & $83-7-10$ & 83 & 10 & 7 \\
\hline
\end{tabular}

La Figura 2 esquematiza los ciclos de sinterización utilizados en ambos métodos pulvimetalúrgicos. Las muestras en verde obtenidas del método CT se sinterizan en un horno eléctrico tubular marca "Sentro Tech" y que posee una bomba de alto vacío marca "Edwards". El ciclo de sinterizado consiste en dos etapas. En primer lugar, se eligen los tiempos y temperaturas adecuados para la descomposición de las partículas de $\mathrm{TiH}_{2}$. Para ello, las muestras en verde se calientan hasta una temperatura de $550{ }^{\circ} \mathrm{C}$ con una permanencia de 90 minutos. El $\mathrm{TiH}_{2}$ es transformado en Ti metálico e $\mathrm{H} 2$ gaseoso, el cual es eliminado. Posteriormente, se lleva a cabo la segunda etapa de sinterización que tiene como finalidad llevar a cabo la unión de las partículas de Ti a través de un proceso difusional en estado sólido. Este proceso es realizado con protección de atmósfera de vacío a $750{ }^{\circ} \mathrm{C}$ por un tiempo de 240 minutos. La temperatura de trabajo se selecciona por debajo de $800^{\circ} \mathrm{C}$ para evitar la reacción de descomposición química de la HAP. Una vez finalizado el ciclo de sinterizado del método CT se obtiene un material compuesto de Ti-HAP.

Por otra parte, las muestras obtenidas con el método GC3D se sinterizan en tres etapas. La primera etapa produce la

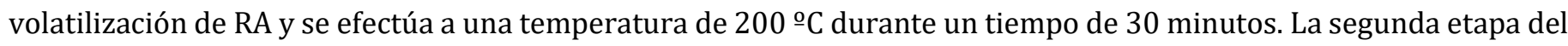
ciclo de sinterización corresponde a la descomposición química de las partículas de $\mathrm{TiH}_{2}$ y se realiza a una temperatura de $600{ }^{\circ} \mathrm{C}$ durante 45 minutos. Por último, la tercera etapa es la sinterización de las partículas de Ti metálico a $780{ }^{\circ} \mathrm{C}$, con una duración de 420 minutos. Una vez finalizado el ciclo de sinterización del método GC3D (Figura 2), se obtiene un material compuesto de Ti-HAP-Poros.

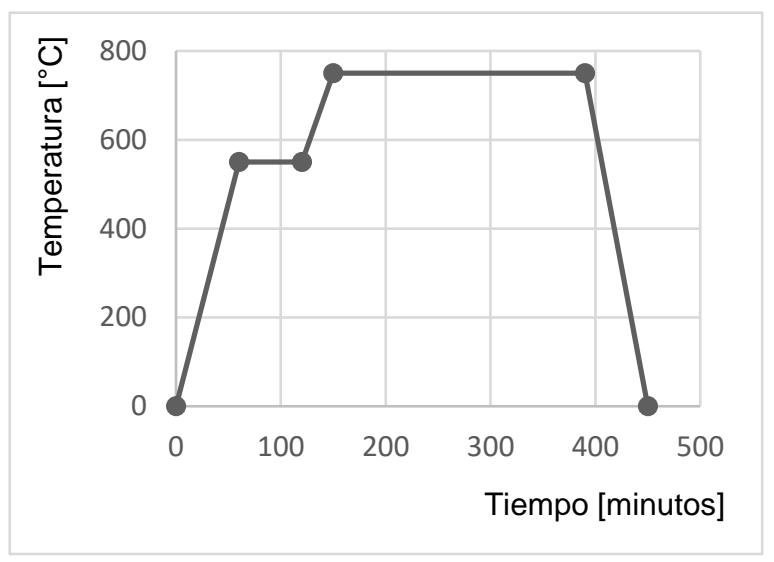

(a)

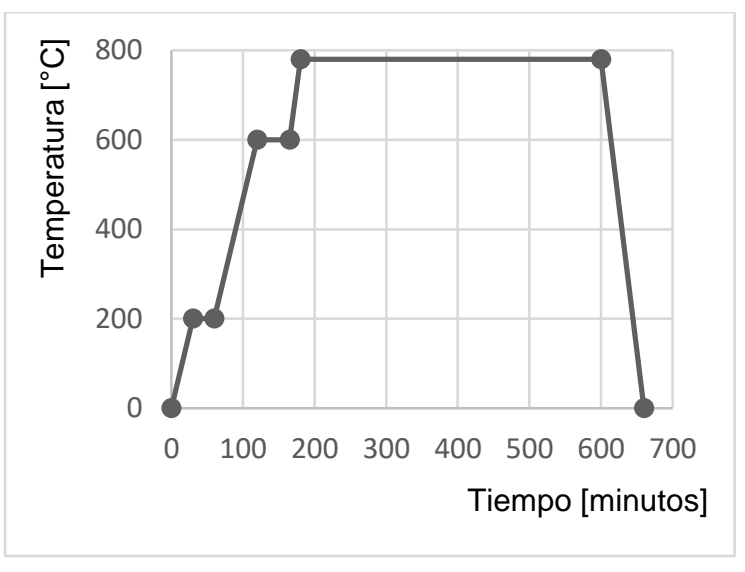

(b)

Figura 2. Esquemas de los ciclos de sinterización utilizados en los métodos pulvimetalúrgicos. (a) CT. (b) GC3D.

\section{Resultados}

\section{Propiedades Mecánicas}

La Tabla 2 muestra los resultados dimensionales en las muestras luego de los sinterizados para los métodos CT y GC3D. Se evidencia la variación de las dimensiones respecto al tamaño inicial de las muestras y las contracciones por sinterización. El método CT muestra porcentajes mayores de contracción volumétrica y podría asociarse a un mejor contacto entre las partículas de $\mathrm{TiH}_{2}$ en las muestras. Asimismo, el método GC3D posibilita la formación de poros en 
su estructura por la volatilización de la RA durante el sinterizado, disminuyendo el contacto entre partículas de $\mathrm{TiH}_{2}$ en las muestras.

Tabla 2. Dimensionales en las muestras luego de los sinterizados en los métodos CT y GC3D.

\begin{tabular}{|c|c|c|c|c|c|c|}
\hline Método & Muestra & $\begin{array}{c}\text { Diámetro } \\
\text { [mm] }\end{array}$ & $\begin{array}{c}\text { Longitud } \\
\text { [mm] }\end{array}$ & $\begin{array}{c}\text { Sección } \\
{\left[\mathrm{mm}^{2}\right]}\end{array}$ & $\begin{array}{c}\text { Volumen } \\
{\left[\mathrm{mm}_{3}\right]}\end{array}$ & $\begin{array}{c}\text { Contracción } \\
\text { volumétrica [\%] }\end{array}$ \\
\hline \multirow{6}{*}{$C T$} & $95-5(1)$ & 7,61 & 6,61 & 45,46 & 300,50 & 25 \\
\hline & $95-5(2)$ & 7,64 & 6,62 & 45,82 & 303,33 & 24 \\
\hline & $90-10(1)$ & 7,79 & 6,63 & 47,64 & 315,83 & 21 \\
\hline & $90-10(2)$ & 7,76 & 6,70 & 47,27 & 316,71 & 21 \\
\hline & $95-15(1)$ & 7,78 & 6,64 & 47,51 & 315,50 & 21 \\
\hline & $95-15(2)$ & 7,60 & 6,96 & 45,34 & 315,58 & 21 \\
\hline \multirow{6}{*}{ GC3D } & $88-7-5(1)$ & 6,65 & 11,35 & 34,71 & 394,01 & 14 \\
\hline & $88-7-5(2)$ & 6,70 & 11,30 & 35,24 & 398,20 & 13 \\
\hline & $86-7-7(1)$ & 6,65 & 11,40 & 34,71 & 395,75 & 14 \\
\hline & $86-7-7$ (2) & 6,65 & 11,30 & 34,71 & 392,28 & 15 \\
\hline & $83-7-10(1)$ & 6,70 & 11,20 & 35,24 & 394,67 & 14 \\
\hline & $83-7-10(2)$ & 6,75 & 11,00 & 35,77 & 393,43 & 15 \\
\hline
\end{tabular}

Una vez efectuado el relevamiento dimensional, se realizaron los ensayos mecánicos y los resultados arrojados se detallan en la Tabla 3. Se observan diferencias sustanciales entre los diferentes métodos de fabricación. A modo de referencia, se deben considerar para los huesos corticales valores de límite de fluencia entre 104 y 224 MPa y módulo de elasticidad entre 10 y $30 \mathrm{GPa}$ y para los huesos trabeculares valores mucho más variables, entre 0,1 y 35 MPa para límite de fluencia y 0,1 a $4 \mathrm{GPa}$ para el módulo de elasticidad. Analizando los resultados obtenidos por el método CT se evidencia valores próximos a los estudiados para el hueso cortical. Para el método GC3D se observan valores más bajos y que se encuentran próximos a los recomendados para los huesos trabeculares. La disminución sustancial de las características mecánicas de las muestras GC3D se puede asociar a la presencia de porosidad en el material compuesto obtenido. Esto también provoca la baja elongación de las muestras.

Tabla 3. Resultados de los ensayos mecánicos en muestras obtenidas por los métodos CT y GC3D.

\begin{tabular}{|c|c|c|c|c|c|c|}
\hline Método & Muestra & $\begin{array}{c}\text { Resistencia a la } \\
\text { compresión [MPa] }\end{array}$ & $\begin{array}{c}\text { Elongación } \\
\text { porcentual [\%] }\end{array}$ & $\begin{array}{c}\text { Módulo de } \\
\text { elasticidad [GPa] }\end{array}$ & $\begin{array}{c}\text { Límite de } \\
\text { fluencia [MPa] }\end{array}$ & $\begin{array}{c}\text { Dureza } \\
\text { [HV] }\end{array}$ \\
\hline \multirow{5}{*}{ CT } & $95-5(1)$ & 749 & 31 & 12,9 & 290 & 115 \\
\cline { 2 - 7 } & $95-5(2)$ & 745 & 32 & 13,8 & 260 & 108 \\
\cline { 2 - 7 } & $90-10(1)$ & 650 & 33 & 9,7 & 156 & 97 \\
\cline { 2 - 7 } & $90-10(2)$ & 628 & 35 & 10,8 & 147 & 99 \\
\cline { 2 - 7 } & $95-15(1)$ & 440 & 34 & 9,3 & 138 & 89 \\
\cline { 2 - 7 } & $95-15(2)$ & 425 & 37 & 9,6 & 124 & 87 \\
\hline \multirow{5}{*}{ GC3D } & $88-7-5(1)$ & 26 & 7 & 0,53 & 2,2 & 25 \\
\cline { 2 - 7 } & $88-7-5(2)$ & 27 & 7 & 0,53 & 2,2 & 25 \\
\cline { 2 - 7 } & $86-7-7(1)$ & 25 & 8 & 0,44 & 2,2 & 22 \\
\cline { 2 - 7 } & $86-7-7(2)$ & 24 & 8 & 0,44 & 2,2 & 23 \\
\cline { 2 - 7 } & $83-7-10(1)$ & 20 & 9 & 0,24 & 2,1 & 21 \\
\cline { 2 - 7 } & $83-7-10(2)$ & 19 & 9 & 0,27 & 2,1 & 20 \\
\hline
\end{tabular}

\section{Conclusiones}

El estudio planteado en este trabajo consistió en evaluar dos métodos pulvimetalúrgicos para obtención de biomateriales compuestos de titanio e hidroxiapatita. Las principales conclusiones que se pueden extraer son:

- Ambos métodos pulvimetalúrgicos llevados a cabo permiten obtener muestras de material compuesto de titanio e hidroxiapatita. 
- El método tradicional de compactación (CT) genera muestras en verde con mayor contacto entre partículas, las cuales se traducen en un incremento de las características mecánicas luego de la sinterización. Por su parte, el método que emplea la técnica de gel-casting y moldes fabricados por impresión 3D (GC3D) produce muestras con cavidades o poros por volatilización de la resina acrílica empleada, conduciendo así a una disminución del contacto entre las partículas y una disminución en las propiedades mecánicas.

- El método CT posibilita obtener valores del módulo elástico y límite de fluencia próximos a los recomendados para los huesos corticales. Por otra parte, el método GC3D produce un material compuesto con valores del módulo elástico y límite de fluencia próximos a los recomendados para los huesos trabeculares de menor densidad ósea.

\section{Referencias}

Lario-Femenía, J., Amigó-Mata, A., Vicente-Escuder, Á., Segovia-López, F., \& Amigó-Borrás, V. (2016). Desarrollo de las aleaciones de titanio y tratamientos superficiales para incrementar la vida útil de los implantes. Revista de Metalurgia, 52(4), 084. https://doi.org/10.3989/revmetalm.084

Duffo, G. (2011). Materiales y materias primas. Biomateriales, guía didáctica.

Traini, T., Piattelli, A., Caputi, S., Degidi, M., Mangano, C., Scarano, A., Perrotti, V., \& Iezzi, G. (2015). Regeneration of human bone using different bone substitute biomaterials. Clinical Implant Dentistry and Related Research, 17(1), 150-162. https://doi.org/10.1111/cid.12089

Padilla, R. L., Lucci, R., \& Oldani, C. (2018). Titanium structures with porosity gradient - Space holder manufacturing and characterization. Revista Materia, 23(2). https://doi.org/10.1590/s1517-707620180002.0422

García-Garduño, M. V., \& Reyes-Gasga, J. (2006). La hidroxiapatita, su importancia en los tejidos mineralizados y su aplicación biomédica. TIP Revista especializada en ciencias químico-biológicas, 9(2), 90-95.

Pereira G, Á., \& Oliva M, P. (2014). Eficacia de la Hidroxiapatita en la Cicatrización de Injertos Óseos e Implantes Dentales: una Revisión Sistemática de la Literatura. International Journal of Odontostomatology, 8(3), 425-432. https://doi.org/10.4067/s0718-381x2014000300017

Londoño, M. E., Echavarría, A., \& De La Calle, F. (2006). CARACTERÍSTICAS CRISTALOQUÍMICAS DE LA HIDROXIAPATITA SINTÉTICA TRATADA A DIFERENTES TEMPERATURAS. In Revista EIA.

Niespodziana, K., Jurczyk, K., Jakubowicz, J., \& Jurczyk, M. (2010). Fabrication and properties of titaniumhydroxyapatite nanocomposites. Materials Chemistry and Physics, 123(1), 160-165.

https://doi.org/10.1016/j.matchemphys.2010.03.076

Segura, N., \& Soria, L. G (2019). Desarrollo de implantes biomédicos base titanio asistidos con impresión 3D (Tesis de Grado). Departamento de Ingeniería Metalúrgica, UTN-FRC, Córdoba, Argentina.

Bocco, F.G; \& Colazo, J. A (2017). Desarrollo de biomateriales base titanio activados con hidroxiapatita (Tesis de Grado). Departamento de Ingeniería Metalúrgica, UTN-FRC, Córdoba, Argentina.

Roure, C. A.; \& Cantero, S. M (Noviembre de 2016). Desarrollo de estructuras porosas para implantes base titanio. $16^{\circ}$ Congreso Internacional de Metalurgia y Materiales SAM-CONAMET 2016. Congreso llevado a cabo en Córdoba, Argentina.

Suárez Castrillón, A. M., Tafur Preciado, W., \& Calderón Nieves, P. R. (2015). Aplicación de herramientas CAD/CAM para el diseño y fabricación de prototipos de moldes de inyección de plásticos. Revista Tecnura, 19(46), 115. https://doi.org/10.14483/udistrital.jour.tecnura.2015.4.a09

Horvath, J., \& Cameron, R. (2017). 3D Printed Science Projects. In 3D Printed Science Projects (Vol. 2). Apress Media LLC. https://doi.org/10.1007/978-1-4842-2695-7 